\title{
Estudo de caso: estrutura de pavimento com aplicação de revestimento flexível na Quadra 407 Sul do município de Palmas (TO)
}

Os pavimentos são caracterizados como estruturas constituídas por diversas camadas de materiais, sobrepostas e distintas, sobre o subleito destinado a resistir e distribuir para o subleito esforços horizontais e verticais, melhorando as condições de tráfego quanto à comodidade e à segurança para o usuário. No entanto, para a escolha do melhor método de execução do pavimento, devem-se conhecer as vantagens e desvantagens de cada processo executivo. Para que isso se torne possível, há a necessidade de projetos compatíveis com o clima e tráfego da região, com a finalidade de analisar as vantagens técnicas, ecológicas, econômicas e sociais, e assim optar pelo projeto mais adequado. O objetivo principal deste trabalho é realizar uma análise de um trecho da pavimentação da Quadra 407 Sul do Município de Palmas (TO) através do método mecanístico. Nesse trabalho, foi utilizada a nova ferramenta disponibilizada pelo DNIT para dimensionamento de pavimentos: $\mathrm{MeDiNa}^{\oplus}$ - Método de Dimensionamento Nacional.

Palavras-chave: Pavimento flexível; Método mecanístico; Palmas (TO).

\section{Case study: pavement structure with flexible coating application in Quadra 407 South of Palmas (TO)}

The pavements are characterized as structures consisting of several layers of materials, overlapping and distinct, on the subgrade intended to resist and distribute horizontal and vertical efforts to the subgrade, improving traffic conditions for convenience and safety for the user. However, to choose the best method of pavement execution, one must know the advantages and disadvantages of each executive process. To make this possible, there is a need for projects that are compatible with the region's climate and traffic, in order to analyze the technical, ecological, economic and social advantages, and thus opt for the most suitable project. The main objective of this work is to perform an analysis of a section of paving of Quadra 407 South of Palmas (TO) through the mechanistic method. In this work, the new tool provided by DNIT for floor sizing was used: MeDiNa ${ }^{\oplus}$ - National Sizing Method.

Keywords: Flexible pavement; Mechanistic method; Clapping (TO)

Topic: Engenharia Civil

Reviewed anonymously in the process of blind peer.
Received:11/01/2019

Approved: 11/03/2019
Erick Phellipe Rodrigues Carvalho

Instituto Tocantinense Presidente Antônio Carlos Porto, Brasi http://lattes.cnpq.br/2799554869225841

erickphellipe23@gmail.com

Flávio Vieira da Silva Júnior

Instituto Tocantinense Presidente Antônio Carlos Porto, Brasil http://lattes.cnpq.br/2211436659738090

flaviovisiju@gmail.com
Referencing this:

CARVALHO, E. P. R.; SILVA JÚNIOR, F. V.. Estudo de caso: estrutura de pavimento com aplicação de revestimento flexível na Quadra 407 Sul do município de Palmas (TO). Engineering Sciences, v.7, n.1, p.30-38, 2019. DOI: http://doi.org/10.6008/CBPC2318-3055.2019.001.0004

DOI: 10.6008/CBPC2318-3055.2019.001.0004 


\section{INTRODUÇÃO}

A história da pavimentação nos remete à própria história, desde a povoação dos continentes, aquisição territorial permuta mercantil, cultura e religião, urbanização e desenvolvimento (FARIA, 2013). Bernucci et al. (2010) define pavimento como uma estrutura de diversas camadas erguida sobre a superfície final de terraplenagem proposta a resistir aos esforços procedentes de um tráfego intenso de veículos, clima e melhoria para os usuários.

Devido às más condições das rodovias brasileiras e o impacto na economia que isso traz em relação a acidentes e desconforto dos usuários, juntamente com os altos preços dos transportes de pessoas e mercadorias, surgem, desse fato, mecanismos e práticas de recuperação de pavimentos flexíveis. De acordo com Bernardes (2013), a maior parte das estradas pavimentadas, no Brasil, são compostas por pavimentos flexíveis, cerca de $95 \%$, os demais são compostos por pavimentos rígidos ou semirrígidos. Assim, há a necessidade de evitar patologias ou recuperar as patologias já existentes.

Com isso, a tecnologia desempenha um importante papel nesse aspecto. Muitos são os debates acerca da melhor metodologia a ser empregada na construção de pavimentação que oferece melhor relação custo benefício. Considerando questões como vida útil, resistência, consumo de material, tempo e a complexidade de execução, custo, impactos ambientais e sociais e ainda a segurança do pavimento durante a demanda do tráfego de veículos (DNIT, 2009).

As principais razões de ocorrência de patologias em pavimentos consistem na baixa capacidade de suporte do subleito, nas irregularidades da superfície de rolamento e na adeficiência do sistema de drenagem. Com isso, problemas como ondulações, afundamentos de trilho de roda, atoleiros, excesso de pó, pista com baixa aderência, costelas de vacas, segregação lateral, buracos e erosões, são comumente encontrados nessas rodovias (FEITOZA, 2014).

Para a escolha do melhor método de execução do pavimento, deve-se conhecer as vantagens e desvantagens de cada processo executivo. Para que isso se torne possível, há a necessidade de projetos compatíveis com o clima e tráfego da região, com a finalidade de analisar as vantagens técnicas, ecológicas, econômicas e sociais, e assim optar pelo projeto mais adequado para a construção da via pavimentada. Nesse panorama, o objetivo principal deste trabalho é realizar uma análise de um trecho crítico da pavimentação pelo método flexível da Quadra 407 Sul, no munícipio de Palmas (TO).

\section{REVISÃO TEÓRICA}

Segundo Balbo (2011), pavimentos são estruturas constituídas por diversas camadas de materiais, sobrepostas e distintas, sobre o subleito destinado a resistir e distribuir para o subleito esforços horizontais e verticais. Assim, o objetivo fundamental do pavimento é resistir, distribuir e repassar ao subleito as cargas solicitadas à via para a qual foi projetada, melhorando as condições de tráfego quanto à comodidade e à segurança para o usuário (SOUZA, 1980). 


\section{Importância do Pavimento}

O deslocamento entre diferentes locais/regiões é realizado através de ruas e estradas, constituindo um elemento urbano que deve ser interpretado como suporte de múltiplos usos, não sendo classificado apenas como um elemento funcionalista para a circulação de veículos e pedestres, mas também como local de relações permanentes entre os usuários proporcionando uma integração social, econômica, cultural e/ou política (JACOBS, 2000).

Uma estrada pavimentada corretamente apresenta a superfície regular e mais aderente aos pneus utilizados nos meios de transporte, e assim, os riscos quanto à perda de controle do veículo são menores garantindo a resposta eficiente a qualquer necessidade de frenagens ou desvios repentinos (GUIMARÃES NETO, 2011). Ainda de acordo com Guimarães Neto (2011), a viagem se torna mais rápida e confortável, o que evita o acúmulo de veículos nas vias e reduz o tempo de deslocamento, acelerando assim a circulação de bens e serviços.

A economia em manutenção, tanto do pavimento, quanto dos veículos utilizados, está entre as principais vantagens ao usuário com relação a uma pavimentação de qualidade, haja vista que o recurso para implantação e manutenção da pavimentação de estradas vem de impostos cobrados do mesmo (GUIMARÃES NETO, 2011).

\section{Pavimento Rígido}

Caracteriza-se o pavimento rígido pela inaptidão à deformação. Como exemplo deste, têm-se os pavimentos constituídos principalmente de concreto de cimento. Esse pavimento é composto pelas camadas de subleito, sub-base e pela placa de concreto com ou sem barras de transferências (figura 1). O revestimento do pavimento rígido possui uma elevada rigidez em relação às camadas inferiores e, portanto, absorve praticamente todas as tensões provenientes do carregamento aplicado conforme afirma o Manual do DNIT (2006).

O Manual de Pavimentos Rígidos (DNIT, 2004) contempla as metodologias aplicadas para a utilização dos materiais de concreto de cimento Portland, os estudos dos concretos, a aplicabilidade do projeto e o dimensionamento, a execução e controle tecnológico de sub-bases e de pavimento, além da conservação e reabilitação do pavimento de concreto.

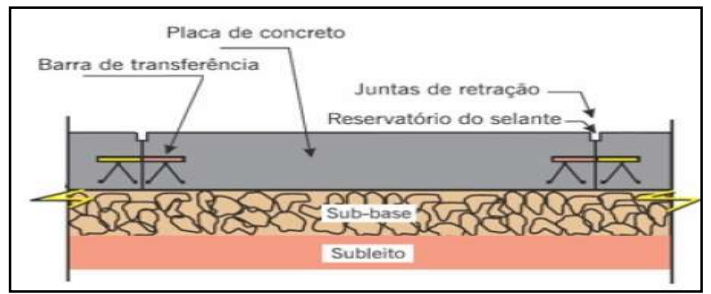

Figura 1: Pavimento rígido com barras de transferência. Fonte: Bianchi et al. (2008).

O pavimento rígido é mais resistente que o flexível e pode durar até 30 anos; assim possui uma grande durabilidade. Além dessa durabilidade maior, testes realizados revelaram que o pavimento de concreto tem uma capacidade de carga maior do que o comum. Em relação aos custos, Balbo (2011) revela 
que ele é cerca de $20 \%$ mais caro, o que seria compensado, no entanto, pela maior durabilidade e pela redução dos custos de manutenção dos veículos e do próprio pavimento de concreto.

\section{Pavimentos Flexíveis}

Pavimento Flexível é composto por revestimento asfáltico sobre camada de base granular ou sobre camada de base de solo consolidado granulometricamente. Os esforços do tráfego são absorvidos pelas distintas camadas representadas da estrutura do pavimento. A estrutura do pavimento é concebida para auferir e imprimir esforços de maneira a aliviar pressões sobre camadas inferiores, mas na maioria das vezes são menos resistentes (BALBO, 2011). O pavimento flexível é formado pelas camadas de reforço de subleito, subleito, sub-base, base e o revestimento asfáltico (figura 2).

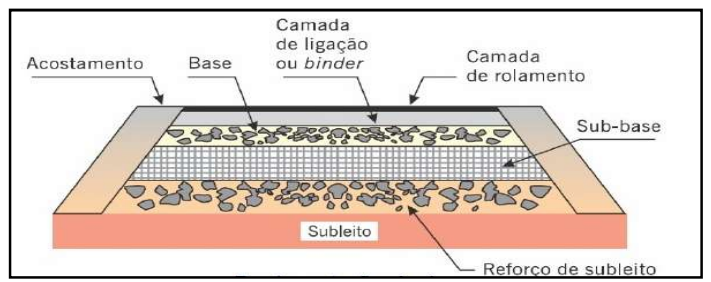

Figura 2: Camadas do pavimento flexível. Fonte: Bianchi et al. (2008).

No pavimento flexível, as cargas são imprimidas à fundação de forma abrandada e também ajuizada, prevenindo a ocorrência de deformações incompatíveis com a utilização da estrutura ou mesmo de rupturas na fundação, que geram estados de tensão não previstos primeiramente nos cálculos e distorcem toda a estrutura a um procedimento mecânico inapropriado e à degradação célere ou prematura (BALBO, 2011).

\section{Métodos de dimensionamento de pavimentos flexíveis}

Existem dois métodos de dimensionamento de pavimentos flexíveis: métodos empíricos e métodos empíricos mecanísticos. Os métodos empíricos se baseiam em fórmulas, experiências e são restritos a pavimentos submetidos a condições idênticas. As aplicações limitam-se ao clima, materiais e condições de carga a que são submetidos. Podem ser os que empregam ensaios de resistência dos solos ou não: Método do Índice de Grupo (IG), método do HRB (HighwayResearchBoard) não empregam resistência dos solos, possuem bons resultados dos ensaios do limite de liquidez, limite de plasticidade e granulometria.

Relaciona propostas de espessuras de camadas com dois grupos de solos: granulares e solos finos. Os métodos empíricos que empregam resistência dos solos é o método do CBR (CaliforniaBearingRatio) e relaciona a capacidade de suporte do subleito e a intensidade do tráfego com a espessura mínima necessária ao pavimento. $\mathrm{O}$ método de Hveem, onde o dimensionamento através desse método considera o efeito destrutivo de deformação do tráfego, a resistência à deformação plástica do solo do subleito e a resistência à tração das camadas constituintes do pavimento.

Para a utilização deste método, é necessária a adaptação dos valores de equivalência utilizados em sua formulação para a região em estudo. Este Método utiliza em seu ensaio equipamentos especiais para a reprodução da compactação. Método empírico mecanístico, por Murillo Lopes de Souza, adaptado do 
Método de dimensionamento de aeroportos do Corpo de Engenheiros dos Estados Unidos (USACE), é baseado em critério de resistência/ruptura ao cisalhamento visa a proteção do pavimento das deformações plásticas excessivas durante a vida útil do projeto; Facilidade de aplicação, comprovada eficácia quando aplicados à realidade brasileira. Considera alguns estudos da pista experimental da AASHO (American AssociationofStateHigwayOfficials), Coeficiente de equivalência estrutural das camadas (K).

Os pavimentos projetados através deste método apresentam grande resistência à ocorrência de deformações permanentes prematuras. Este método, roteiriza o dimensionamento de pavimentos flexíveis em função da capacidade do subleito (CBR) e índice de grupo IG e do número equivalente de operações do eixo padrão (N) e espessura total do pavimento durante um período de projeto (ALBANO, 2013).

A atuação da mecânica dos pavimentos está na análise de tensões, deformações e deslocamentos na estrutura do pavimento, sendo estes conhecidos como parâmetros de deformabilidade. As análises destes parâmetros são, normalmente, realizadas através de programas computacionais, como é o caso do programa $\mathrm{MeDiNa}^{\circledR}$.

\section{METODOLOGIA}

Esse estudo foi realizado na cidade de Palmas, capital do Tocantins, situado na região central do Estado. A área territorial da capital é de $2.218,943 \mathrm{~km}^{2}$ o que corresponde a $0,80 \%$ da totalidade do Estado (IBGE, 2014). O trecho analisado neste estudo corresponde a uma parcela da área de pavimentação de aproximadamente $182.000 \mathrm{~m}^{2}$ que possui cerca de $26.000 \mathrm{~m}$ de drenagem. Localizado na Quadra 407 Sul, mostrado na Figura 3, na região central da capital sob as coordenadas geográficas de $10^{\circ} 20^{\prime} 67.70^{\prime \prime} \mathrm{S}$ e $48^{\circ}$ $35^{\prime} 31.5016^{\prime \prime} \mathrm{O}$, sua altitude corresponde a 504m do nível do mar.

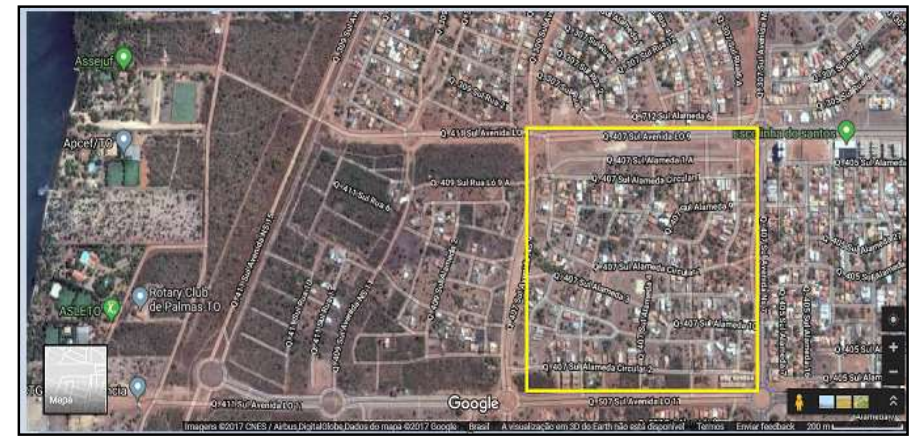

Figura 3: Localização da área de estudo.

Os dados foram coletados de acordo com o memorial descritivo da obra, os resultados da obra foram realizados e fornecidos por empresa de engenharia que preferiu não se identificar. Realizou-se a comparação dos resultados do projeto de pavimentação utilizada na obra com os resultados obtidos a partir dos cálculos de Módulo Resiliente, que foram obtidos a partir de dados prévios do ensaio CBR de cada camada (subleito, base e sub-base). Para o dimensionamento de cada uma das camadas foi utilizado o software $\mathrm{MeDiNa}^{\circledR}$ do DNIT (Método de Dimensionamento Nacional). Para este trabalho, foi considerado apenas um trecho de 200 a 300 metros da quadra que será escolhido em decorrência da maior movimentação, conforme o volume diário de veículos (VMD). 


\section{Estimativa do Volume de Tráfego}

A estimativa do Volume de Tráfego (ou Fluxo de Tráfego) foi elaborada com base nas determinações ditadas pelo DNIT (2006), onde se calcula o número de veículos que passam por uma seção de uma via, ou de uma determinada faixa, durante uma unidade de tempo, normalmente, expresso em veículos/dia (vpd) ou veículos/hora (vph).

Os autores completam que a média da quantidade de veículos que circulam num período de 24 horas em um trecho de via é caracterizado com VMD. Assim, foi computado para um período representativo, o qual, salvo indicação em contrário, é de um ano. A quantidade de veículos, que melhor representa a utilização ou serviço prestado pela via, foi usada para indicar a necessidade de novas vias ou melhorias das existentes, estimar benefícios esperados de uma obra viária, determinar as prioridades de investimentos, calcular taxas de acidentes, entre outros.

O volume de tráfego inclui todos os veículos que circulam pela via em um só sentido ou em ambos, ou ainda, os que circulam por uma só faixa. O conhecimento do VDM de uma determinada via é imprescindível para a formulação de medidas seja de execução da pavimentação asfáltica, seja a elaboração de medidas ou restauração do pavimento. Com esse valor pode-se determinar o dimensionamento e as possíveis variações das camadas do pavimento. A obtenção dos dados do volume de tráfego foi disponibilizada pela empresa responsável pela construção do pavimento analisado.

\section{Método para Dimensionamento dos Pavimentos Flexível}

Para o dimensionamento pelo Módulo Resiliente, através do software computacional MeDiNa do DNIT, usa-se como base o valor do ensaio CBR do solo utilizado em cada camada, para base e sub-base foram utilizados solos transportados de uma jazida próxima a Palmas (TO), os ensaios de CBR portando foram realizados e fornecidos pela empresa responsável pela jazida. Então se adotou, para base CBR 60, sub-base CBR 40 e subleito CBR 11 (lembrando que para o subleito foi utilizado o próprio solo da região, uma argila siltosa). Equação utilizada para determinação de módulo resiliente a partir do CBR foi: MR=326 + $67 *$ CBR) Resultado em $\mathrm{kgf} / \mathrm{cm}^{2}$ convertido para MPa para ser utilizado no software.

\section{RESULTADOS E DISCUSSÃO}

O projeto estudado nesta pesquisa contempla também obras de melhoria da infraestrutura no município de Palmas (TO), visando à prevenção, o controle e a minimização dos impactos provocados pelas enchentes urbanas, através da macrodrenagem das quadras, pré-requisito para pavimentação dela.

Na figura 4, está apresentado no material descritivo pré-determinado pela empresa responsável, onde as obras foram executadas no Plano Diretor Central da cidade de Palmas, beneficiando a população residente nas quadras que sofrem a intervenção direta, bem como, das circunvizinhas. Na figura, percebe-se que o dimensionamento que foi realizado na obra analisada, apresenta as camadas de base e sub-base com uma espessura de $20 \mathrm{~cm}$ cada, sobre o subleito regularizado e compactado, material do subleito constando 
o próprio solo natural da região, apresentando uma argila Siltosa com valor de CBR 11, para camada de base CBR 60, sub-base CBR 40. Com base nos dados coletados e conhecimento das características do tráfego da região e da cidade de Palmas, adotou-se como veículo padrão aquele cuja carga é de 8,2T por roda para um tráfego muito leve/leve para o interior das quadras, com N=1x10 ${ }^{4}$, caracterizado como Tráfego Médio.

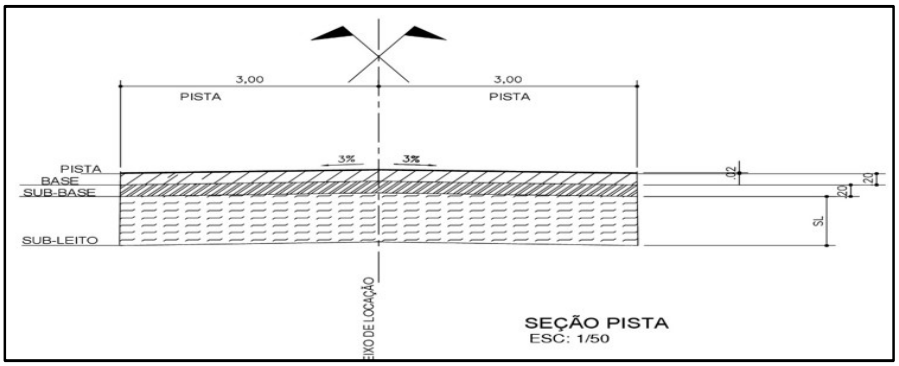

Figura 4: Dimensionamento do pavimento conforme o projeto da obra analisada.

No entanto, os resultados mostraram que há um subdimensionamento na estrutura do pavimento, uma vez, que a espessura das camadas de base e sub-base deveriam ter, respectivamente, uma espessura de 20 e 40cm de acordo com o sistema MeDiNa de Dimensionamento Nacional, para uma margem confiável de execução do pavimento, como se observa na figura 5.

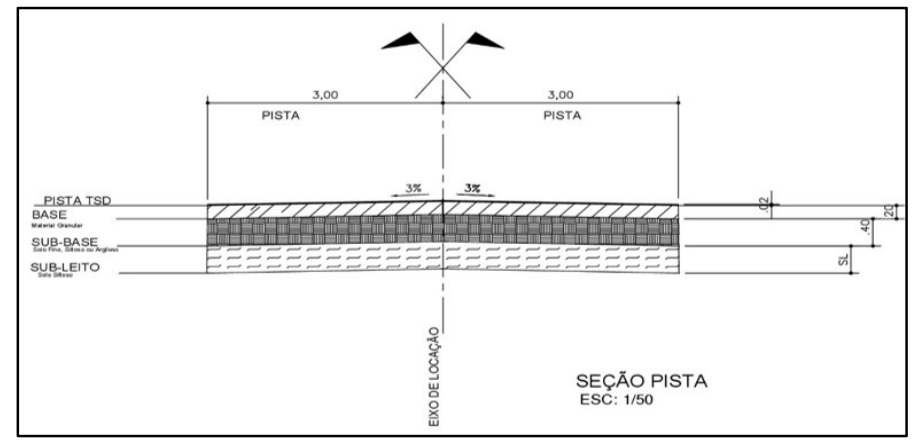

Figura 5: Dimensionamento do pavimento conforme o Software.

A justificativa da diferença entre os valores executados e os apresentados pelo software ocorre devido aos diversos parâmetros utilizados pelo software para o dimensionamento de pavimentos, como a resiliência do material utilizado em cada camada, bem como o clima da região que corrobora para a danificação precoce do pavimento. A tabela 1 apresenta os dados obtidos por meio do software, conforme cada camada do pavimento.

Tabela 1: Dimensionamento do pavimento baseado no material e espessura.

\begin{tabular}{|c|c|c|c|c|}
\hline Camada & Material & Espessura $(\mathbf{c m})$ & Módulo de Resiliência & Coeficiente de Poisson \\
\hline 1 & $\begin{array}{c}\text { TRATAMENTO SUPERFICIAL } \\
\text { Tratamento Superficial Duplo }\end{array}$ & 2,0 & $\mathrm{MR}=1000 \mathrm{MPa}$ & 0,25 \\
\hline 2 & $\begin{array}{c}\text { MATERIAL GRANULAR } \\
\text { Base Palmas }\end{array}$ & 20,0 & $\mathrm{MR}=426 \mathrm{MPa}$ & 0,35 \\
\hline 3 & $\begin{array}{c}\text { SOLO FINO, SILTOSO OU ARGILOSO } \\
\text { Sub-Base Palmas }\end{array}$ & 40,0 & $\mathrm{MR}=295 \mathrm{MPa}$ & 0,35 \\
\hline 4 & SUBLEITO Solo Siltoso NS' & $\mathrm{SL}$ & $\mathrm{MR}=189 \mathrm{MPa}$ & 0,45 \\
\hline
\end{tabular}

O material utilizado na execução do pavimento foi transportado de uma jazida próxima ao município de Palmas (TO). Após a realização do ensaio CBR efetuou-se o cálculo do da equação ("MR=326+67*CBR") 
para converter o resultado de $\mathrm{kgf} / \mathrm{cm}^{2}$ para MPA. Assim, as análises realizadas com o software aumentam a precisão dos resultados para o dimensionamento adequado para atender com segurança e precisão o requisito de durabilidade do pavimento a ser executado.

Tabela 2: Definição do tráfego conforme o software.

\begin{tabular}{|c|c|}
\hline \multicolumn{2}{|l|}{ DEFINIÇÃO DO TRÁFEGO } \\
\hline Volume Médio Diário no ano de abertura do tráfego: VMD (1ํ ano) & 27 \\
\hline Fator de veículo no ano de abertura do tráfego: FV & 1,00 \\
\hline Número de passagens anual do eixo padrão (1ㅇano) & $1,00 \mathrm{e}+04$ \\
\hline$\%$ Veículos na faixa de projeto & $100 \%$ \\
\hline Número de passagens anual do eixo padrão na faixa de projeto & $1,00 \mathrm{e}+04$ \\
\hline Taxa de crescimento do tráfego: & $2,5 \%$ \\
\hline Número Equivalente total de passagens do eixo padrão na faixa de projeto: $\mathrm{N}$ Eq & $5,25 e+04$ \\
\hline
\end{tabular}

\section{CONCLUSÕES}

Com a utilização do método empírico de dimensionamento dos pavimentos, muitos parâmetros importantes para a condição de vida útil do pavimento são descartados. Dessa forma, o método de dimensionamento mecanístico de pavimento vem para aprimorar a técnica de dimensionamento bem como melhorar a condições dos pavimentos brasileiros. Com a utilização do software MeDiNa, o dimensionamento do pavimento ganha credibilidade e deve melhorar consideravelmente as condições de rolamentos dos locais onde forem utilizados. Ao demonstrar que o método de dimensionamento mecanístico aponta um subdimensionamento da sub-base, como visto, traz à tona que o método empírico para o atual tráfego impostado ao Brasil, não atende de forma satisfatória os requisitos básicos para um pavimento durável e de qualidade.

\section{REFERÊNCIAS}

ALBANO, J. F.. Hierarquia e classificação funcional de vias rurais e urbanas: Tópicos avançados em vias rurais e urbanas. Porto Alegre: UFRGS, 2013.

BALBO, J. T.. Pavimentação asfáltica: materiais, projeto e restauração. São Paulo: Oficina de Textos, 2011.

BERNARDES, D. J.. Recuperação de pavimentos flexíveis em áreas de tráfego pesado devido a implantação de lombadas eletrônicas. Uberlândia: UNITRI, 2013.

BERNUCCI, L. B.; MOTTA, L. M. G.; CERATTI, J. A. P.; SOARES, J. B.. Pavimentação Asfáltica: formação básica para engenheiros. 3 ed. Rio de Janeiro: Imprinta, 2010.

BIANCHI, F. R.; BRITO, I. R. T.; CASTRO, V. A. B.. Estudo comparativo entre Pavimento rígido e flexível. Vitória: UCL, 2008.

DNIT. Departamento Nacional de Infraestrutura dos Transportes. Manual de pavimentos rígidos. 2 ed. Rio de Janeiro: DNIT, 2004.
DNIT. Departamento Nacional de Infraestrutura dos Transportes. Manual de Pavimentos Flexíveis: Publicação IP-714. 2 ed. Rio de Janeiro: DNIT, 2009.

DNIT. Departamento Nacional de Infraestrutura dos Transportes. Projeto de norma e manuais. Rio de Janeiro: DNIT, 2006.

FARIA, E. O.. História dos transportes terrestres no mundo. Rio de Janeiro: UFRJ, 2013.

FEITOZA, E. C.. Análise patológica e técnicas de recuperação de pavimentos flexíveis em vias urbanas. Monografia (Graduação em Engenharia Civil) - Universidade Federal do Piauí, Teresina, 2014.

GUIMARÃES NETO, G. L.. Estudo Comparativo entre a Pavimentação Flexível e Rígida. Monografia (Graduação) - Universidade da Amazônia, Belém, 2011.

IBGE. Instituto Brasileiro de Geografia e Estatística. Cidades. Rio de Janeiro: IBGE, 2014. 
JACOBS, J.. Morte e vida de grandes cidades. São

Paulo: Martins Fontes, 2000.

SENÇO, W.. Manual técnico de pavimentação. São

Paulo: Pini, 2001.
SOUZA, M. L.. Pavimentação Rodoviária. Rio de Janeiro: DNER, 1980.

A CBPC - Companhia Brasileira de Produção Científica (CNPJ: 11.221.422/0001-03) detém os direitos materiais desta publicação. Os direitos referem-se à publicação do trabalho em qualquer parte do mundo, incluindo os direitos às renovações, expansões e disseminações da contribuição, bem como outros direitos subsidiários. Todos os trabalhos publicados eletronicamente poderão posteriormente ser publicados em coletâneas impressas sob coordenação da Sustenere Publishing, da Companhia Brasileira de Produção Científica e seus parceiros autorizados. Os (as) autores (as) preservam os direitos autorais, mas não têm permissão para a publicação da contribuição em outro meio, impresso ou digital, em português ou em tradução. 